\title{
Evaluation of Airborne Precision Spacing in a Human-in-the-Loop Experiment
}

\author{
Dr. Bryan E. Barmore* \\ National Aeronautics and Space Administration, Langley Research Center, Hampton, VA 23681 \\ Terence S. Abbott ${ }^{\dagger}$ \\ Booz-Allen Hamilton, Hampton, VA 23681 \\ William R. Capron ${ }^{\ddagger}$ \\ Lockheed Martin Corporation, Hampton, VA 23681
}

\begin{abstract}
A significant bottleneck in the current air traffic system occurs at the runway. Expanding airports and adding new runways will help solve this problem; however, this comes with significant costs: financially, politically and environmentally. A complementary solution is to safely increase the capacity of current runways. This can be achieved by precisely spacing aircraft at the runway threshold, with a resulting reduction in the spacing buffer required under today's operations. At NASA's Langley Research Center, the Airspace Systems program has been investigating airborne technologies and procedures that will assist the flight crew in achieving precise spacing behind another aircraft. A new spacing clearance allows the pilot to follow speed cues from a new on-board guidance system called Airborne Merging and Spacing for Terminal Arrivals (AMSTAR). AMSTAR receives Automatic Dependent Surveillance-Broadcast (ADS-B) reports from an assigned, leading aircraft and calculates the appropriate speed for the ownship to fly to achieve the desired spacing interval, time- or distance-based, at the runway threshold. Since the goal is overall system capacity, the speed guidance algorithm is designed to provide system-wide benefits and stability to a string of arriving aircraft.

An experiment was recently performed at the NASA Langley Air Traffic Operations Laboratory (ATOL) to test the flexibility of Airborne Precision Spacing operations under a variety of operational conditions. These included several types of merge and approach geometries along with the complementary merging and in-trail operations. Twelve airline pilots and four controllers participated in this simulation. Performance and questionnaire data were collected from a total of eighty-four individual arrivals. The pilots were able to achieve precise spacing with a mean error of 0.5 seconds and a standard deviation of 4.7 seconds. No statistically significant differences in spacing performance were found between in-trail and merging operations or among the three modeled airspaces. Questionnaire data showed general acceptance for both pilots and controllers. These results reinforce previous findings from full-mission simulation and flight evaluation of the in-trail operations. This paper reviews the results of this simulation in detail.
\end{abstract}

\section{Nomenclature}

$\begin{array}{ll}\text { ADS-B } & \text { Automatic Dependent Surveillance - Broadcast } \\ \text { AMSTAR } & \text { Airborne Merging and Spacing for Terminal Arrivals } \\ \text { APS } & \text { Airborne Precision Spacing } \\ \text { ATAAS } & \text { Advanced Terminal Area Approach Spacing } \\ \text { ATC } & \text { Air Traffic Control }\end{array}$

*Aerospace Engineer, Aviation Operations and Evaluation Branch, 1 South Wright Street, MS 156A. AIAA member. ${ }^{\dagger}$ Sr. Research Engineer, 1 South Wright Street, MS 156A, Langley Research Center

${ }^{\ddagger}$ Sr. Research Scientist, Space Operations, 1 East Ames Street, MS 460, Langley Research Center 


$\begin{array}{ll}\text { ATOL } & \text { Air Traffic Operations Laboratory } \\ \text { CDTI } & \text { Cockpit Display of Traffic Information } \\ \text { CNS } & \text { Communication, Navigation and Surveillance } \\ \text { FAF } & \text { Final Approach Fix } \\ \text { FMS } & \text { Flight Management System } \\ \text { MCDU } & \text { Multi-function Control Display Unit } \\ \text { NAS } & \text { National Airspace System } \\ \text { PDS } & \text { Pair-Dependent Speed } \\ \text { PFD } & \text { Primary Flight Display } \\ \text { RNAV } & \text { Area Navigation } \\ \text { STAR } & \text { Standard Terminal Arrival Route } \\ \text { TRACON } & \text { Terminal Radar Approach Control } \\ \text { VNAV } & \text { Vertical Navigation }\end{array}$

\section{Introduction}

During the 1990's, air travel increased at an unprecedented rate, placing ever increasing capacity pressures on the National Airspace System (NAS). While the events of 2001 temporarily relieved these pressures, there are clear signs of returning demand and gridlock. It is important to continue to address these capacity issues so that future U.S air transportation growth is not impeded. As part of this effort, numerous government and industry efforts are underway to develop new procedures for airborne and ground-based concepts to increase the capacity of the NAS.

With a focus on improving terminal area arrival capacity, NASA has developed a concept called Airborne Precision Spacing (APS). APS provides a means for merging multiple in-bound streams and delivering precisely spaced aircraft to the runway threshold. A qualified flight crew on a properly equipped aircraft is able to use speed guidance cues and advanced displays to merge behind and space relative to an ATC-specified aircraft.

Previous research has indicated the feasibility of using traffic information on the flight deck to enable airborne-managed spacing. ${ }^{1-9}$ Early simulator experiments conducted at NASA Langley concluded that time-based spacing was the most promising technique for precision spacing. Time-based spacing allows for the natural distance compression between aircraft to occur while they slow during an arrival. Accurate knowledge of the position and speed of the lead aircraft with fast update rates are necessary for concept feasibility. Recent advancements in computing power and the initial deployment of Automatic Dependent Surveillance-Broadcast (ADS-B) make this concept realizable.

Airborne Precision Spacing evolved from one part of NASA's Advance Air Transportation Technologies (AATT) project. The activity, called Self-Spacing for Terminal Arrivals, was part of the Distributed AirGround Traffic Management activity ${ }^{\mathrm{a}}$. Initial implementation focused on in-trail spacing with the goal of reducing the variability of the inter-arrival spacing at the runway threshold, thereby increasing runway throughput. The air traffic controller would issue a strategic clearance for an aircraft to follow a designated aircraft and to achieve the assigned spacing interval at the runway threshold. The crew would then follow the on-board speed guidance provided by the Advanced Terminal Area Approach Spacing (ATAAS) tool to precisely achieve the spacing. Monte Carlo studies ${ }^{10}$ showed the possibility for capacity increases with resulting significant reductions in potential arrival delays without changing any other operating procedures. High-fidelity simulations ${ }^{11,12}$ and a flight test ${ }^{13}$ verified that this new operation could achieve the desired precision and accuracy without any negative impact on crew workload. Studies on a similar concept by Eurocontrol ${ }^{14,15}$ have shown a decrease in controller workload and reduced radio communications.

Following the success of the ATAAS flight test, it was decided to extend the capabilities to include merging traffic streams. This extension would allow for the spacing clearance to be issued earlier in the arrival and allow more time to achieve the assigned spacing. To use ATAAS, the two aircraft had to already be in-trail before the spacing clearance could be implemented. The earlier the operation can begin, the lower the radio congestion will be and the less precisely the aircraft need to be delivered to the point where spacing

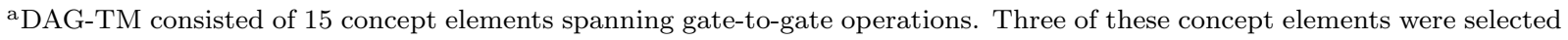
for initial study and development. This one, concept element 11, was the only select element focusing on terminal arrival productivity. 
would begin. Within operationally acceptable speed variations, an aircraft can adjust it's relative position by approximately $1 \mathrm{~nm}$ in every $20 \mathrm{~nm}$ flown.

The ATAAS tool was expanded and renamed AMSTAR (Airborne Merging and Spacing for Terminal Arrivals). In this concept, incoming aircraft are assigned RNAV routes from terminal area entry to ILS intercept. These routes consists of a lateral path and a nominal vertical and speed profile. The route is used as a reference trajectory by the spacing tool. AMSTAR takes the current position of the lead aircraft as reported over ADS-B and computes the leading aircraft's time to the threshold from its reference trajectory. The same is done for the ownship; the difference in the estimated times of arrival at the runway threshold is compared to the assigned spacing value, yielding a spacing error. The ownship's speed is then adjusted relative to this spacing error. Several refinements are also made to promote the acceptability for both the crew and the controller and the stability of the overall operations. These include notch filtering, gain scheduling and speed limiting. For more details see Ref. 16.

\section{Experiment Design}

Since aircraft and flight crews are not restricted to just a few arrival airports, it is important that any airborne technology and associated procedures be designed with little to no site-specificity. Also, since each airport has it's own unique constraints on airspace design, it is important that Airborne Precision Spacing be readily adaptable to a wide variety of route designs. To this end, we designed an experiment that would investigate the flexibility of the APS tools and associated procedures to a variety of realistic arrival routes and merge geometries. Several representative, high-demand US airports were simulated to "host" this experiment based on the geometries and characteristics of their approach paths. This airport selection was designed to provide a basis for examining a range of merging angles, distances of the merge point to the final approach fix (FAF), angles of the descent path from the TRACON entry, and lengths of the final approach path. Only a portion of the selected airports' airspace was reproduced to support the requirements of the experiment; many operational considerations were neglected. For example, the interaction and proximity of other traffic, going to either alternate runways at the selected airport or nearby satellite airports, was not modeled. This was considered to be more of an airspace design issue than a fundamental issue for the flexibility of the concept and will be studied in future experiments. The airspaces used were Northern California (NorCal) TRACON, Chicago TRACON and New York TRACON with traffic arriving at LaGuardia.

All arrival routes were defined as FMS arrival routes and were based on published STARs or RNAV procedures that were extended to intercept the ILS course. The goal was to make a straight-forward extension of currently used arrival procedures and connect them with approach procedures to create a continuous route from TRACON entry to the runway. In places where all segments of the routes were not defined, input was sought from pilots familiar with the airspace operations. In only one case was there a need to create a new waypoint. That was in the Chicago airspace where there was not an existing waypoint where the turn from inbound to downwind legs for one of the routes.

All altitude crossing restrictions were based on published data for the route. Where available, speed reduction points were also taken from published procedures. Otherwise, our consulting pilots assisted in ensuring a reasonable speed schedule. All scenarios started with the aircraft just outside the TRACON entry point.

The first selected geometry consisted of a standard entry leg leading to a downwind leg. The second route would then merge at either the turn to base or turn to final. The aircraft would turn on to the final approach course approximately 15 miles from the runway threshold. To model this type of airspace we selected Chicago O'Hare. Arrival routes for runway 22R were established for aircraft entering on either the BEARZ or TADDS FMS route. See figure 1 for details. Both of these routes cover approximately $60 \mathrm{~nm}$ from the starting point to the runway threshold. This allows for a shallow descent angle averaging $1.5^{\circ}$ until the glideslope intercept. There is $12 \mathrm{~nm}$ from NOLAN, where the BEARZ arrivals intercept the final approach course, to the runway and $19 \mathrm{~nm}$ from FNUCH where the TADDS arrivals turn onto final. There is a $90^{\circ}$ intercept between the two routes at NOLAN.

The second arrival route geometry chosen was one where the two merging routes have a shallow intercept angle. This has the effect of stretching out the merging region but also lessens the impact of the turn dynamics of the aircraft. A good example of this type of operations are two arrivals from the south and southwest of New York going into LaGuardia's runway 4. We developed the KORRY and BEUTY FMS routes (see figure 2). Both routes are approximately $60 \mathrm{~nm}$ long. The arrival via KORRY is nearly a straight 


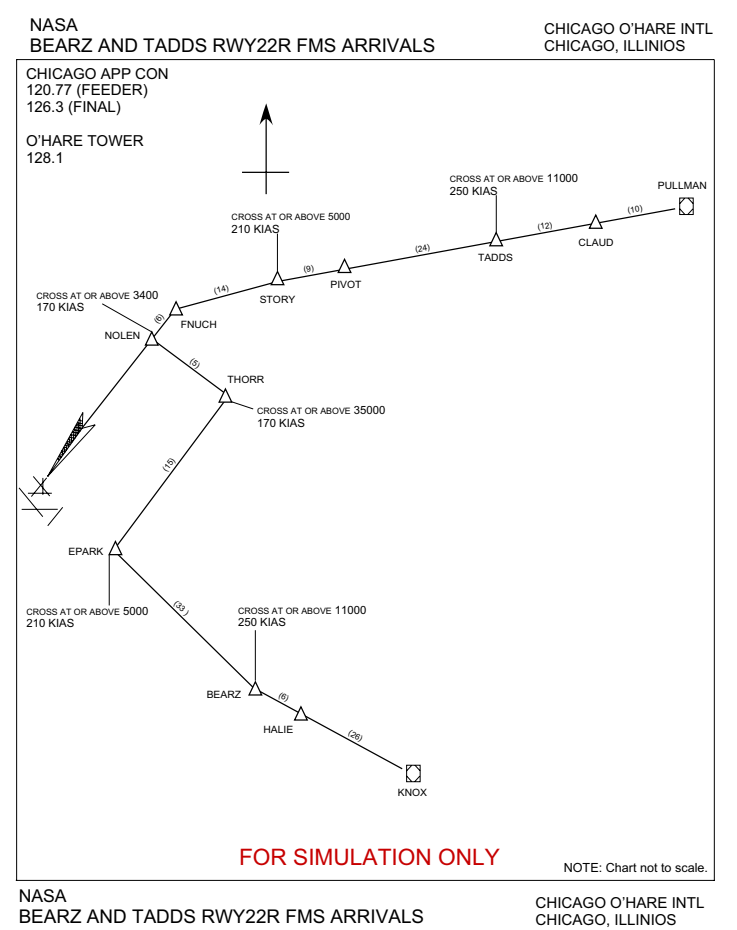

Figure 1. Arrival routes for Chicago TRACON used in this experiment.

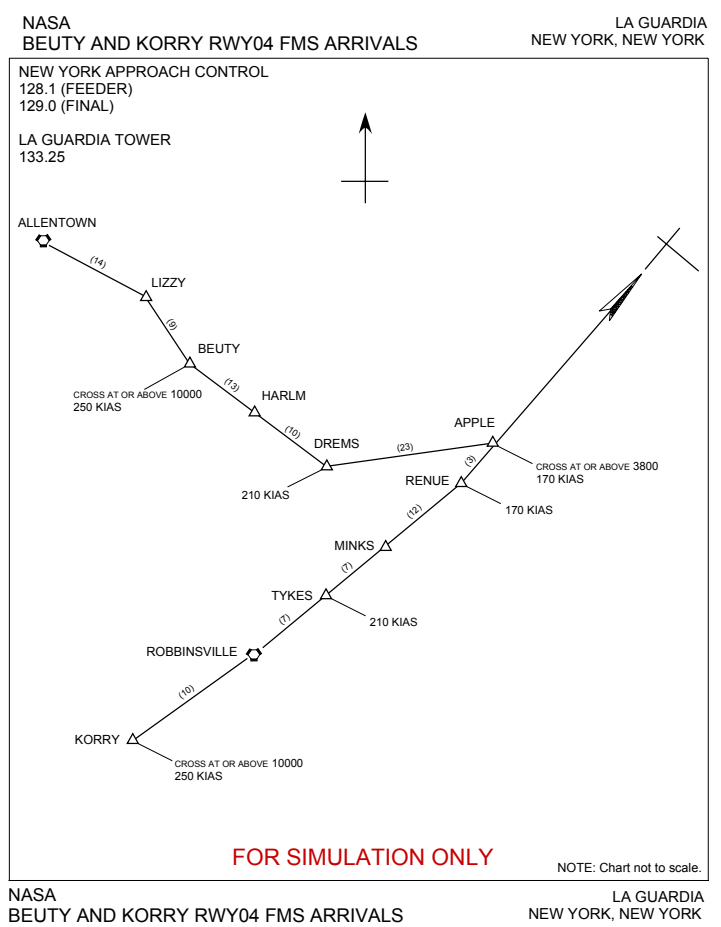

Figure 2. Arrival routes for New York TRACON used in this experiment.

shot while the BEUTY arrival has several turns in it as it dips around Newark Liberty International Airport airspace. The two routes merge at approximately a $30^{\circ}$ angle. It was nearly $17 \mathrm{~nm}$ from APPLE, the merge point, to the runway.

The final geometry we were interested in had a steep descent that would make managing a deceleration at the same time difficult. We had also wanted to look at a case where the aircraft would have a rather short combined final approach segment. Both of these are found in the San Francisco airspace. We chose the CEDES and SKUNK FMS routes leading to runway 28R. Based on terrain and other air traffic considerations, the arriving aircraft are kept high until late in the arrival. On both routes, there were descents of between $7000 \mathrm{ft}$ and $8000 \mathrm{ft}$ over a $22 \mathrm{~nm}$ flight path. This gives a flight path angle of $3^{\circ}$ to $3.4^{\circ}$. During this time, the aircraft must also be decelerating from $250 \mathrm{kt}$ to $170 \mathrm{kt}$. This caused the pilots to actively manage their aircraft's drag. The approach chart is shown in figure 3 .

The aircraft arriving via CEDES would turn onto final approach at DUMBA which is $14 \mathrm{~nm}$ from the runway threshold. Those arriving via SKUNK would not join the final course until CEPIN which is less than $10 \mathrm{~nm}$ from the threshold. This greatly limited the region where traffic was actually in-trail. Soon after crossing CEPIN, all aircraft would start the deceleration to their final approach speed. So spacing needed to be nearly achieved before the merge point in this case. The two routes converge at about a $50^{\circ}$ angle at CEPIN.

In all cases there was a nominal wind field included that was a constant 10 knots directed 10 degrees east of the run-

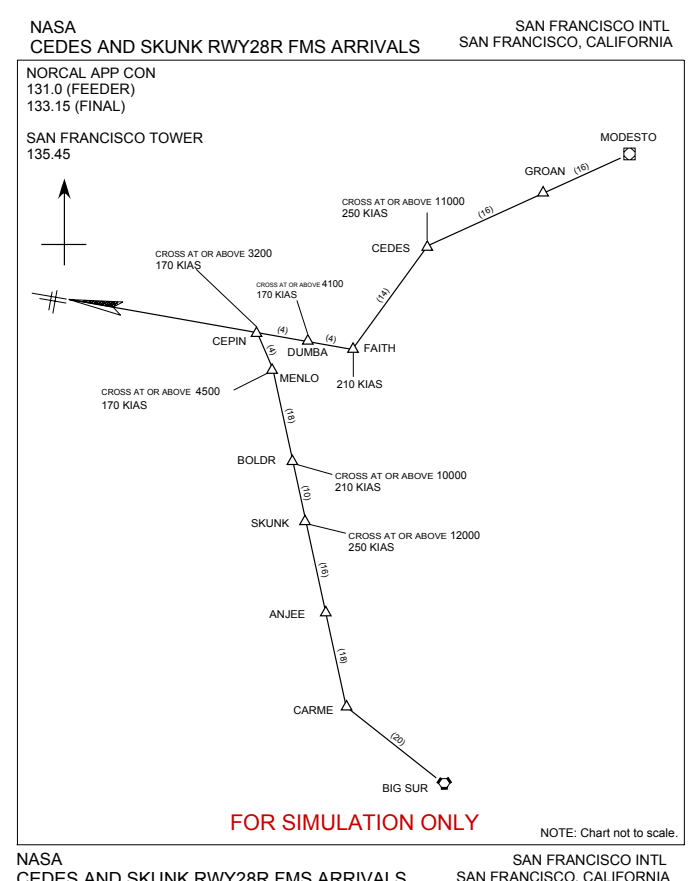

Figure 3. Arrival routes for NorCal TRACON used in this experiment. 
way alignment. A predicted wind field of 12 knots and 20 degrees east of the runway alignment was used to simulate wind prediction errors. An additional real-life uncertainty was added by delivering the aircraft to the TRACON entry normally distributed around the scheduled time with a 15 second standard deviation.

A $3 \times 2$, within-subjects experiment design was used with the arrival airspace and type of operations, merging or in-trail, as the independent variables. The type of operation was choosen to see if there were any impacts to performance introduced by the extension to merging traffic. The procedures and speed guidance algorithm for aircraft in-trail were nearly identical to the previous work done with the ATAAS tool. An additional baseline run was performed; this was used to seperate out difficulties with the simulation environment from difficuties with the new procedures. Each scenario consisted of six subject pilots, two subject controllers and three confederate pilots. One confederate pilot lead the stream and flew the standard profile without any spacing clearance. The subject pilots were active line pilots from major commercial or cargo airlines with recent experience with Boeing glass cockpits. The controllers were active controllers from a range of medium to hub-sized TRACONs. Two sessions were run involving a total of 12 subject pilots and four controllers. The scenarios seen were the same between the two sessions. All subjects were here on personal time and not as part of their normal duties.

The experiment was performed in NASA Langley's Air Traffic Operations Laboratory (ATOL). The ATOL houses twelve medium-fidelity, workstation-based flight deck simulators; a high-fidelity future communication navigation surveillance (CNS) infrastructure; a target generator; and several low-fidelity controller workstations. The flight deck simulator, called ASTOR, models current basic aircraft components include: aircraft and engine models; autopilot and autothrottle systems; flight management computer (FMC) and multi-function control display unit (MCDU); mode control panel (MCP) and electronic flight instrumentation system (EFIS) control panel; displays such as the primary flight display (PFD), navigation display (ND), and engine indication and crew alerting system display (EICAS); sensor systems; and the communications management unit (CMU). Current advanced technology components include: representations of the future CNS (ADS-B); and on-board systems that manage traffic information, flow constraint information, and airspace constraint information. ${ }^{17,18}$

The pilots were currently rated on a modern, Boeing glass cockpit aircraft type such as the 777, 747-400, $757 / 767$ or 737 . Six of the pilots were currently active, four were furloughed and two retired. The majority had 757/767 experience; only one had 777 experience. The pilots were at Langley for two days consisting of half a day of training including several practice runs, seven data runs, and a debriefing session.

The training sessions consisted of briefings on the concept of operations, the use of the ASTOR simulator, and the use of AMSTAR and the pair-dependent speed (PDS) mode. PDS is a new speed guidance mode added to ASTOR that takes the AMSTAR speed guidance and supplies it to the auto-pilot system, replacing the FMC speed guidance when activated. Following both the ASTOR and the AMSTAR/PDS briefing, the pilots were taken into the ATOL where they had hands-on training and several practice sessions.

The ASTOR training focused on the use of the desktop simulator including mouse interactions, the translation of physical knobs and dials to graphical representations, and unique features or behavior of the simulation environment. Attention was paid to known differences between the ASTOR simulator and the behavior of the real aircraft such as VNAV modes, aerodynamic characteristics and flap deployment schedules. It is clear from the data analysis that either additional specific training was needed or the subject pool should have been limited to those with experience on the Boeing 777 .

The AMSTAR/PDS training introduced the pilot interfaces and display modifications as well as Airborne Precision Spacing procedures. Possible failure modes and alerts were presented along with the expected pilot response. Two practice runs were then flown. All pilot training was done in DFW airspace so as to not skew the pilots' performance in the data collection airspaces.

The pilots were instructed to follow all clearances given by the controllers, accept the spacing clearance, engage the AMSTAR tool, and follow the speed guidance provided by AMSTAR. They then monitored the speed and ensured their aircraft was properly configured for landing. The pilot's data run ended after crossing the runway threshold but before touchdown. The pilots had the flexibility to either follow the vertical path using the FMS's vertical navigation (VNAV) or with "flight level change" (FLCH) as they felt comfortable or as their individual company policies recommended. Objective performance data was collected for the aircraft and the pilots completed a questionnaire following each data run.

The controllers were brought in to assist in issuing clearances and evaluating the traffic behavior. The controllers were given a computer display that showed the airspace sectors, the traffic, and the active arrival routes. Each aircraft had an enhanced data tag that showed the assigned spacing and the callsign of their 
lead aircraft. The controllers could also display trend vectors for each aircraft. No additional tools were available to assist in monitoring the spacing conformance. The scripted sequence of aircraft along with the expected arrival route were given to the controllers before each data run. These resembled paper scripts where the controllers could make notes.

Two controller positions were defined: a feeder position that controlled the aircraft from TRACON entry to close to the merge point and a final position that included the merge point and the final approach. For realism, a tower controller position was staffed by a confederate controller, who had no influence on the behavior of the pilots or other controllers. The feeder controller would clear the arriving aircraft for the arrival route and then issue the precision spacing clearance. This clearance took the form of: "American 123, Cleared Precision Spacing, Maintain One Two Zero Seconds Spacing, Reference Continental 321." This would clear American 123 to merge, if necessary, and space 120 seconds behind Continental 321. For this experiment, all aircraft were spaced at 120 seconds which corresponds to a minimum spacing distance that is slightly greater than the required wake vortex separation. As the aircraft approached the sector boundary, the feeder controller would hand them off to the final controller and issue a frequency change to the pilot.

The final controller would take the aircraft as they approached the merge point and then issue a clearance for the ILS approach as the aircraft neared the turn on to the final course. Once established on the localizer and glideslope, the final controller would transfer the aircraft to the tower controller who would clear them to land.

Although the controllers were asked to monitor the behavior of the traffic and judge the conformance and safety of the operations, they were asked not to intervene. Although intervention would be a part of the complete concept, limiting the controller's intervention allowed for more rigorous data analysis. Both subject controllers had the ability to mark an event on their screen and capture the current traffic position for later discussion instead of intervening. The controllers only expressed concern in two instances.

The controllers all had experience from TRACON facilities. The controllers responded to a short questionnaire at the end of the data runs. No performance data was collected or analyzed for the controllers.

\section{Pilot Interfaces and Procedures}

Only a few modifications to the flight deck displays and controls were needed to integrate AMSTAR into the ASTOR simulation. These can be broken down into three types of changes: situation awareness, AMSTAR guidance and AMSTAR interaction. The clearance to begin spacing operations contained two key pieces of information: the reference aircraft and the assigned spacing interval. The pilot would start by going to a new set of MCDU pages that allowed for inputs to the AMSTAR tool. The pilot could select their reference aircraft from a list of all aircraft within ADS-B range. Once the reference aircraft was selected, the pilot would enter the spacing interval (see figure 4). If the reference aircraft was found, AMSTAR would go into armed mode; otherwise, AMSTAR would go into profile mode until the reference aircraft was located. If five minutes passed and the reference aircraft was not located, the

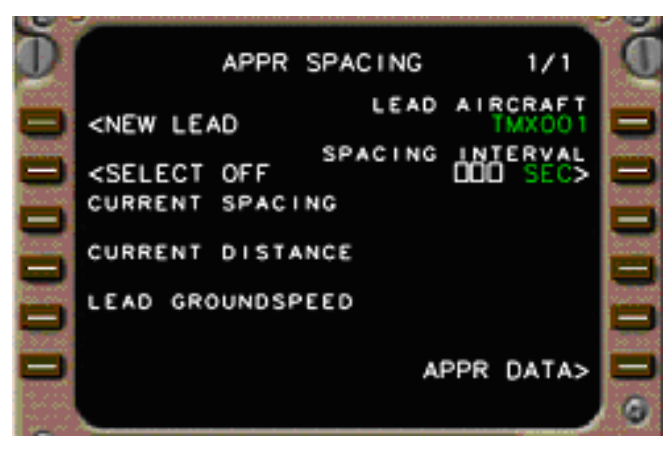

Figure 4. The ASTOR Multi-function Control Display Unit (MCDU) showing the input page for AMSTAR. pilot would be alerted. The pilot would then recheck that they selected the correct aircraft and then contact ATC for further instructions. AMSTAR profile mode is a mode that follows the published speed profile and does not consider any other aircraft. It could be used when the ownship does not have an aircraft in front of it. Profile mode is also used while waiting to receive enough information on the reference aircraft to start actively spacing.

The typical mode for AMSTAR operations is the paired mode. In this case, AMSTAR is receiving enough information on the lead aircraft to calculate the projected spacing at the runway threshold and then offer speed guidance to the crew to meet the assigned spacing. To minimize workload impacts and promote stability in a long stream of aircraft, several criteria are considered before changing the commanded speed. These include the current spacing value, the aircraft configuration, and the reference speed for the current segment. Speed changes come in five knot increments except on the final leg where one knot changes are used to achieve the desired precision. For more details on how AMSTAR works refer to Refs. 16,19. A third 
mode is called "final" and occurs when AMSTAR stops guiding based on the lead aircraft or the profile and slows the aircraft to its programmed final approach speed. While AMSTAR remains engaged for final approach, it does not provide active spacing guidance inside the final approach fix.
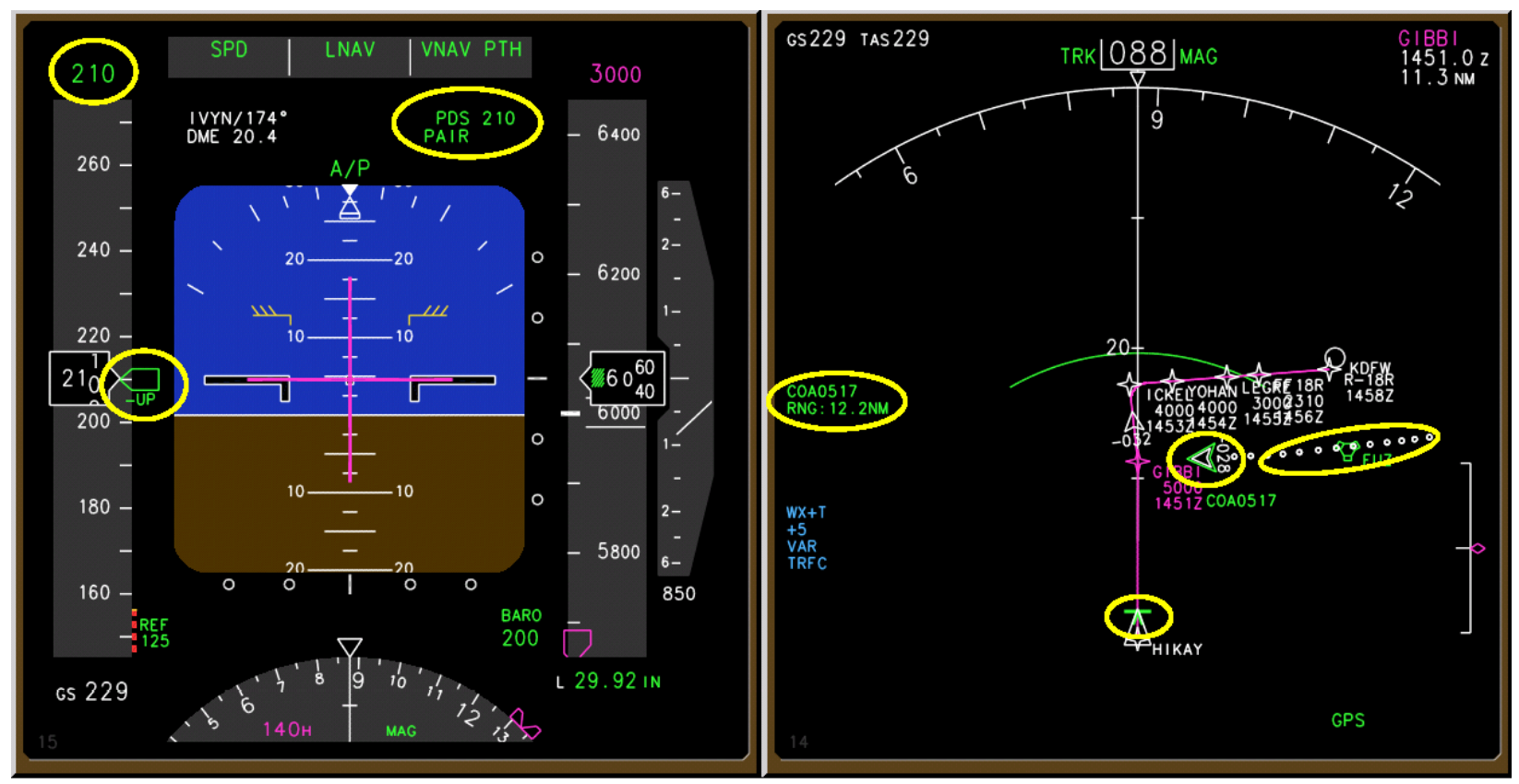

Figure 5. The ASTOR Primary Flight Display (PFD) and Navigation Display (ND). All new symbology is circled in yellow: on the PFD this includes the green text in the upper right and the green speed target and speed bug; on the ND it includes the spacing position indicator just in front of the ownship, the highlighted lead aircraft, the history dots for the lead aircraft and the text block on the left hand side.

AMSTAR mode and guidance information were placed on the Primary Flight Display (PFD) for this experiment, although alternative locations have been considered for nearer-term implementations. AMSTAR status and mode information appears as a small text block toward the upper right corner (see figure 5). The text is white for armed and green for active. The text shows the current mode (PROF, PAIR, FINAL) and the current speed target. When AMSTAR is the active source of speed guidance, the speed target and bug on the speed tape are green. The pilot was able to select AMSTAR as the source of speed guidance using a new mode control button placed on the mode control panel. This new speed guidance mode was called pair-dependent speed (PDS).

The navigation display (ND) (figure 5) was used to show traffic information and provide situation awareness for the spacing operation. The reference aircraft is highlighted with a green outline. A small text block appears on the middle of the left side showing the callsign of the reference aircraft and their slant distance range. At small enough map range settings, a series of history dots appear behind the reference aircraft. These dots show the lateral path flown by the reference aircraft. These are particularly useful for a "follow the leader" operation that was not tested in this experiment. The final addition to the ND was a spacing position indicator (SPI) that showed where AMSTAR was guiding the aircraft to. If the aircraft was aligned with the SPI it would be at the profile speed and predicted to achieve the assigned spacing. The pilots were instructed to follow the speed guidance presented on the PFD and use the ND information only for situation awareness.

Several alerts and messages were available to notify the crew of abnormal or unexpected events during the spacing operations. The most common and relevant for this experiment was a "PDS DRAG REQUIRED" message which appeared when the aircraft was more than 5 knots above the commanded speed and not closing or more than $400 \mathrm{ft}$ above the vertical profile. In these cases, the flight crew would generally need to use speed brakes to regain the speed or the vertical path. This generally occurred in situations where the aircraft was descending near idle thrust and a speed reduction was required. As expected, the use of speed brakes varied depending on the design of the arrival route. 


\section{Experiment Results}

The results will be discussed in three parts: first, the objective aircraft performance; second, performance observations made during the data runs and while replaying the data; and third, the results of both the controller and pilot questionnaires.

\section{A. Performance Data}

As mentioned earlier, the goal of the spacing operation is to precisely space aircraft across the runway threshold. Therefore, the spacing interval was measured as the time difference between consecutive aircraft crossing the threshold. All aircraft were assigned a spacing interval of 120 seconds. As a reminder, the aircraft flew through a wind field that was inaccurately predicted and arrived at the entry point with a spread of times around the scheduled time.

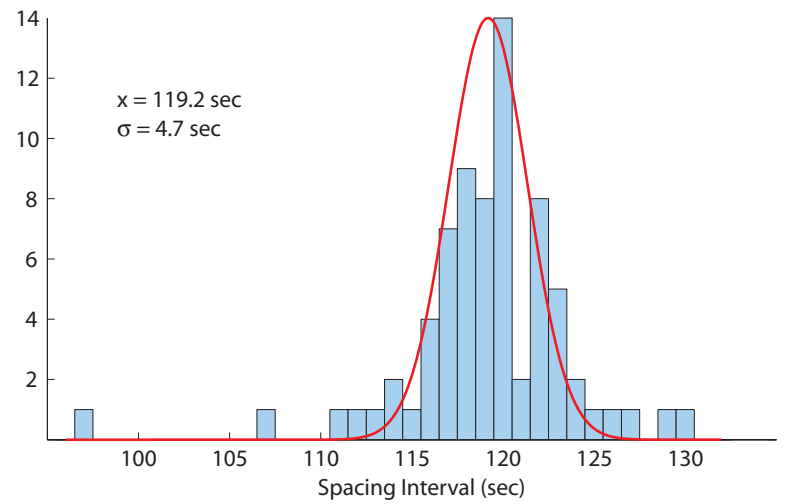

Figure 6. Interarrival spacing results for all subject pilots. Bins are 2 seconds wide. Assigned spacing for all runs was 120 sec.

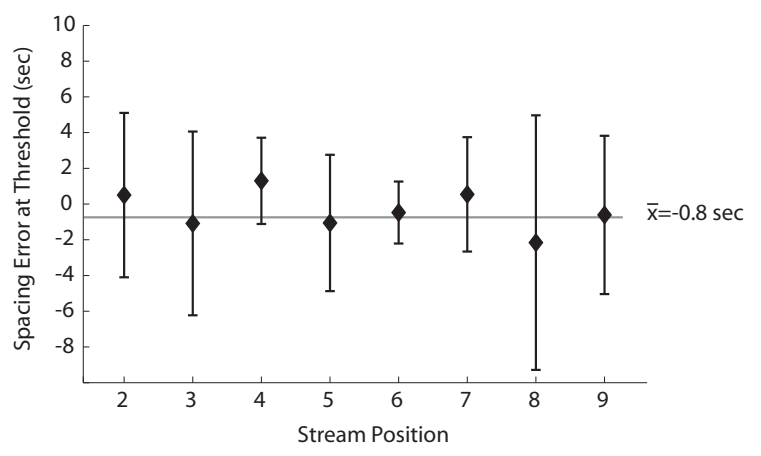

Figure 7. Average spacing error and standard deviation at the runway threshold as a function of position in the string.

Across all conditions, the measured interarrival spacing was $119.2 \pm 4.7$ seconds (mean \pm standard deviation). The standard error was 0.5 seconds. The standard deviation was larger than expected based on previous simulations ${ }^{11}$ where the pilots were able to achieve precision of \pm 2 seconds. As will be shown below, much of this larger spread is attributable to significant flight deviations resulting from the pilots' understanding of the ASTOR simulator. In fact, if the two most extreme outliers, both of which had significant flight deviations, are removed, the standard deviation is reduced to 3.5 seconds.

Figure 6 shows the distribution of interarrival spacing: the goal was $120 \mathrm{sec}$. It is close to a normal distribution with a few outliers, particularly on the low side. The experiment was designed to determine if the performance of spacing operations were effected by the airspace design or the type of operation performed: merging or in-trail. A three-way ANOVA found there to be no statistically significant differences between airspace, operation or subject ( $p>0.60$ for all cases). However, these three sources only accounted for $15 \%$ of the total variation observed. The experiment was not designed to uncover the other sources of variation although significant variations were seen between runs by the same subject pilot.

One of the concerns of the spacing operation is the long-term stability of a string of spacing aircraft. Recent fast-time studies have looked at the effects on strings as long as 100 aircraft, over three hours of operations, and have not seen any destabilizing effects. ${ }^{20}$ Figure 7 shows the spacing error and standard deviation by string position for this experiment. Again, no noticeable instabilities are seen.

To determine the number of additional speed changes, the speed commands issued by AMSTAR were counted. These were generally 5-10 kt speed changes. The speed changes associated with the STAR, generally $30-40 \mathrm{kt}$, were not included in the counting, yielding an average of $5.9 \pm 2.6$, which is consistent with the results found for an in-trail only study in a full mission simulator. ${ }^{11}$ Figure 8 shows that there was no effect due to the position in the string. An ANOVA test showed that the number of speed changes was not dependent on the arrival route, the type of operation or the subject ( $p=0.16,0.79,0.98$ respectively).

As a final look at the overall behavior of an string of aircraft, the running spacing errors during one run are shown in figure 9. This was the second run for the second group in the simulated O'Hare airspace. The spacing error is the difference between the predicted spacing at the threshold and the assigned spacing value 


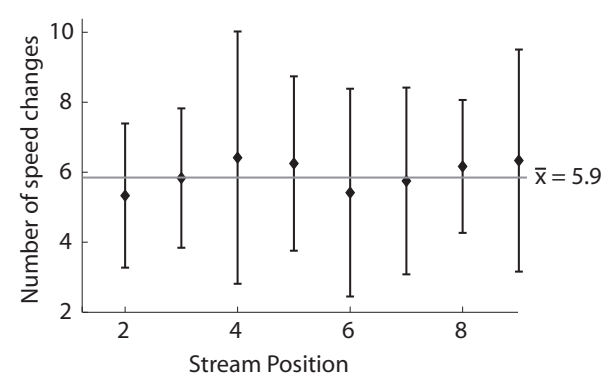

Figure 8. Average number of additional speed changes and standard deviation as a function of position in the string.

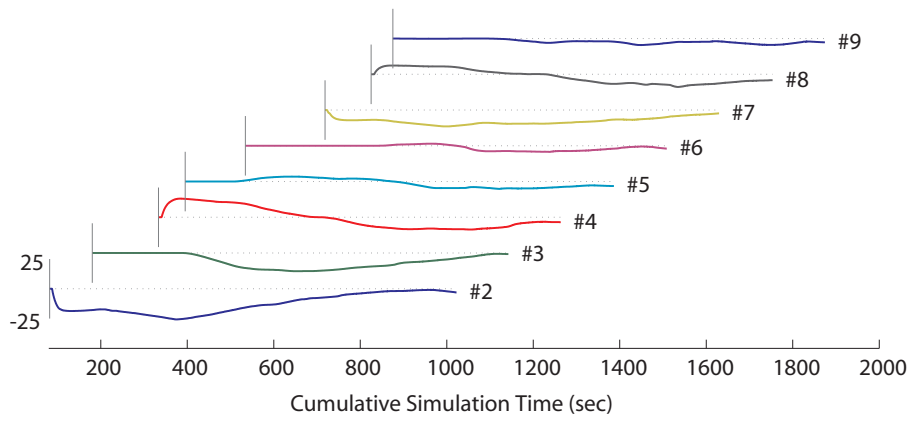

Figure 9. The running spacing error, as calculated on-board, for each aircraft during the second run in ORD airspace for the second experiment group.

and is continually calculated; a negative value means the aircraft is predicted to cross too far behind at the threshold. As seen on the figure, aircraft \#2 starts up behind by about 20-25 seconds. Over time, it slowly recovers by flying 5-10 kt above the profile speed. Aircraft \#3 started well and fell a little behind because its lead was faster than the profile. Aircraft \#4 started ahead and slowed slightly to recover. This pattern continues on through the rest of the stream. No one is ever more than 25 seconds off, and most of the time the spacing error is 10 seconds or less.

\section{B. Performance Observations}

In addition to the analysis of the recorded data, video playback of the pilots actions and interactions with the simulator was analyzed. It was found that there were several confounding effects in the experiment that can be attributed to pilot behavior and training that were unrelated to the spacing operations.

Each pilot performed a baseline run where they followed the FMS path without any spacing operations. Many problems seen during the spacing operations also appeared during these baseline operations suggesting the cause was not the spacing operations or procedures but other effects. It was found that $12 \%$ of the approaches resulted in either an unstable instrument approach or a flaps overspeed. For the baseline runs, that error rate was $10 \%$ and once corrected for the airspace used for the baseline, the operational error rate was identical. While a overall primary cause could not be identified, the problems seemed to be related to:

- poor understanding of B-777 operational procedures (e.g., flap speeds);

- poor understanding of the B-777 VNAV function;

- "slam dunk" arrival procedures (e.g., flap overspeed at SFO) and pilot unfamiliarity with these procedures;

- poor understanding of energy management procedures;

- unrealistic flight deck cues (level of simulator fidelity);

- inadequate simulator or aircraft-specific training (learning effects were seen for several pilots); and

- single pilot operation in a traditional two-crew environment.

Some of these are related to the fidelity of the simulation environment, which suggest that studies like this one need to be validated in higher fidelity simulations. The benefit of studies such as the current one is that the interactions of many piloted aircraft can be investigated at low-cost. Additional problems can be attributed to the pilot pool requirements and training. Half of the pilots had some moderate to severe mode-confusion or misunderstanding problems. The primary case was the autoflight system making an uncommanded transition into a VNAV ALT (vertical navigation with altitude hold) or an ALT HOLD (altitude hold) mode. This occurred when the pilot delayed in selecting the appropriate descent altitude in the MCP altitude window. This is a behavior of the 777 systems but is not seen in current 737 and $757 / 767$ aircraft. It is clear that not enough attention was paid to this and other differences during pilot training. Limiting the selection pool to only 777 pilots would have also helped to eliminate this problem. 
There were additional problems with energy and speed management. Large speed errors were noted in several of the runs. Considering that the AMSTAR guidance typically commanded speed changes at $0.5 \mathrm{kt} / \mathrm{s}$, the large speed errors, many in excess of $20 \mathrm{kt}$, occurred over large intervals of time. Several pilots failed to add drag when needing to decelerate while descending, and instead tried to control the aircraft's energy by changing VNAV modes. The speed discrepancy would cause the aircraft to gain on the leading aircraft and pull away from the trailing aircraft. Since several of the arrival paths required aggressive energy management to maintain proper speed and altitude, incorrect or inadequate use of the airplane's drag devices quickly put these pilots into situations from which recovery was difficult.

It was also noted that all of the subject pilots, at least on the first run, allowed the airplane to accelerate away from the planned speed at the initiation of the glideslope intercept. That is, if the airplane were in level flight prior to glideslope intercept, the descent onto the glideslope would require the immediate application of flaps or landing gear to maintain the speed. Any delay in configuration deployment would allow the airplane to accelerate away from the planned speed. Figure 10 shows the profile speed, actual flown speed and AMSTAR guided speed for two different aircraft during the same run. In the upper figure, we see that the pilot was able to follow the AMSTAR guidance quite well, deviating only slightly near the transition from $225 \mathrm{kt}$ to $170 \mathrm{kt}$. During the early

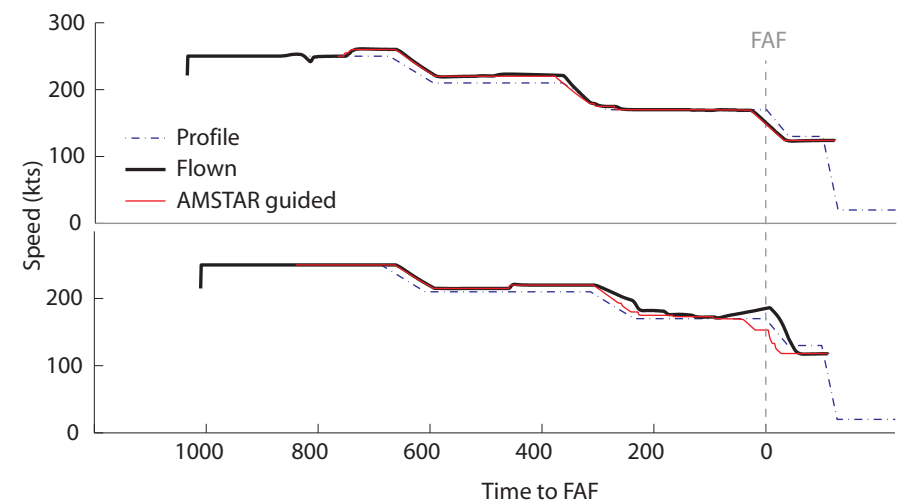

Figure 10. Comparison of the speeds of a well behaved and an illbehaved flight. In the upper figure, the thin, red line showing the actual indicated air speed follows the AMSTAR guidance (thick, black line) almost exactly. In the lower figure, there is a significant deviation with the actual speed being quite a bit faster than the guidance just before the FAF. See text for a more detailed analysis.

part of the run there is a 10-15 kt difference between the speed guidance and the profile. This shows that the aircraft started at more that 120 seconds behind their lead and needed to catch up. That was achieved nearly 200 seconds before the FAF where the guidance and profile coincide. We also see that the final approach speed for this aircraft was about $10 \mathrm{kt}$ slower than the nominal value in the profile. The lower half shows a pilot that had significant speed deviations close to the FAF. Starting at the deceleration point to $170 \mathrm{kt}$ the aircraft is slow in decelerating causing a positive speed deviation. At about 100 seconds before the FAF the glideslope was intercepted and the aircraft started to descend but without adequate drag to maintain speed. The pilot had speedbrakes retracted, flaps 5 and gear up at this point. Between this point and the FAF the aircraft accelerated to $185 \mathrm{kt}$ and the pilot made no changes to the aircraft configuration. The AMSTAR speed was $155 \mathrm{kt}$ and was limited by the aircraft configuration. AMSTAR had issued a caution that additional drag was required but this was not acted upon. Just inside the FAF the pilot was alerted that they we approaching the minimum safe distance behind the lead aircraft. This alert is displayed on the ND and caused the pilot to react. The pilot was able to slow the aircraft to its final approach speed just before crossing the threshold. In a real operation, this would clearly be unacceptable and the controller would have had to intervene. It appears that the pilot lost attention to the simulation during the last two minutes before the FAF. No obvious cause was identified but this could be attributed to the fidelity of the simulation or the fact that this was the last data run for this pilot.

There was only one problem identified that was particular to the use of AMSTAR. In this instance, the pilot seemed to be confused by the speed guidance given when AMSTAR was first engaged. When the spacing clearance was given, the aircraft already had a negative spacing error and thus commanded a speed slower than what the aircraft was currently flying. The pilot seemed to expect AMSTAR to maintain the current speed and tried twice to reinitialized AMSTAR before accepting the slower speed. This all occurred over about 20 seconds. There were no further problems for this pilot.

approximately ten seconds with an initial condition such that the actual spacing was significantly closer than the assigned. Because of this, the initial spacing command was $235 \mathrm{kt}$, which was $15 \mathrm{kt}$ below the nominal STAR speed. Either because the pilot did not understand that the speed was slower than the STAR or did not understand that AMSTAR moderated the reduction in the spacing error, the pilot repeatedly 
tried to engage PDS speed guidance, then selected speed intervention, and then deselected and reselected PDS guidance twice. These actions occurred over a 30 second period. After that time, the pilot seemed to accept the PDS speed guidance.

Due to the large number of problems seen with the non-AMSTAR operational errors, future studies should be done with a more stringent pilot selection process and a more in-depth and thorough training program that includes more focus on the basic simulated airplane and its systems. Results should also be validated in a high-fidelity simulation.

\section{Questionnaire Data}

The general analysis of the questionnaire ratings was centered on the mean ratings for all subjects for a particular question, and for all ratings given by each subject for a particular run. The post-scenario questionnaires that the pilots answered tried to assess their thoughts about the AMSTAR tool and the precision spacing concept. Questions looked at issues such as the level of attention needed for each phase of flight; the pilot's frustration level and assessment of their success in meeting the spacing goal; and the acceptability and usability of the speed guidance and information presented. There were also two open-ended questions where the pilots could give additional feedback. All answers were to be limited to the scenario just finished.

At the end of the experiment, pilots and controllers were asked to complete a post-experiment questionnaire. They were asked several questions about the conduct of the experiment as well as training. The pilots were then asked to rate the overall usefulness and reliability of the tools and procedures. The controllers were asked about the acceptability of the operations and if they thought these operations could be integrated into today's traffic flow. The controllers were also asked to offer real-world situations or constraints that they thought would affect these operations.

All rating questions were based on a seven-point scale with 7 being the most positive and 1 being the most negative answer. The questions on success, frustration and attention were broken down by phase of flight.

In general, the responses were overwhelmingly positive. For the attention, frustration and success questions, average responses were above 6.0. The vast majority of responses to all questions, among all subjects, had ratings of 5 or greater with 7 representing nearly half of all ratings.

The average rating of a particular question among all subjects generally provided insight into how well that run went. For instance, a high rating (average of 6 or higher) for a question regarding the level of attention required to follow the spacing procedure would indicate that most subjects found that the level of attention required was acceptable for that run. We found an example of this at SFO, where due to the steeper descent angle, subjects had to more closely manage energy in order to stay on the target speed. For questions regarding frustration and performance, some subjects gave low average responses to the level of frustration perceived (higher frustration), and low ratings of their own performance in flying the approach, for the middle and final portions of the approach.

On the other hand, the average rating for a particular subject for a run would provide insight into the subject's overall success for that run. Generally speaking, if a subject rated all components of one question unusually low, they rated all or many components of other question unusually low as well. For instance, subjects that rated the "attention required" as high, also rated frustration as high, evaluation of their own performance as low, and usefulness of AMSTAR guidance and other information sources as low.

This experiment was not, nor was it intended to be, an accurate measurement of controller workload nor AMSTAR's effect on it. In a real-world environment, controllers would have other non-equipped aircraft to control, transient aircraft, weather, coordination and many other activities to tend to. So in the absence of these other tasks, and because of the high level of success of the AMSTAR tool in keeping aircraft in the arrival flow properly spaced, the controllers actually were left with little else to do. Thus, many of the controller ratings of the concept, the observation of the experiment airspace and procedures, and the progress of the precision spacing aircraft along the arrival route were extraordinarily high. They did however provide some very useful insight on questionnaire items where they were asked to provide comments and not rating values.

One question asked the controllers what real-world factors not represented in this simulation they thought would hinder airborne merging and spacing operations. Responses included pilot and controller resistance to change, dynamic weather (thunderstorm activity and the re-routing it may require, widely variable winds between velocity, direction, and altitude etc.), and re-sequencing go-arounds and unscheduled arrivals. Other 
concerns raised included the AMSTAR application and procedures associated with arrival routes that have transitions to multiple runways.

However, the comments made about the concept in general were quite positive. They include:

- "As an active controller, having worked this, I hate to go back to the old (present) way."

- "The concept is a much needed tool to increase efficiency of the NAS."

- "I believe the overall efficiency of the system can be greatly increased with this equipment and these procedures."

\section{Conclusion}

Airborne Precision Spacing offers the ability for the flight crew to precisely achieve an ATC-specified inter-arrival spacing interval behind a designated lead aircraft. This increased precision and predictability offers ATC a new tool to reduce workload and improve airport capacity. This experiment was designed to look at some basic feasibility issues associated with flight crew spacing and merging. Strings of nine arriving aircraft were simulated in a variety of airspaces with both merging and in-trail operations. The overall results were positive and support the feasibility of these types of operations.

Twelve subject pilots and four controllers were brought in to a medium-fidelity, single-pilot research lab. Although there were some basic training and familiarity problems with the simulator that may have confounded the results, the overall results were positive. The pilots were able to meet the assigned spacing value with a standard error of 0.5 seconds and a standard deviation of 4.7 seconds. There were an average of 5.9 additional speed changes beyond those listed in the STAR. This was not effected by the place in the string. There was no statistically significant effect on the spacing or the number of speed changes based on the type of operation, airspace or subjects. No destabilizing effects were seen and the effects of the poorly behaving aircraft did not propagate back more than one or two aircraft. Responses from both the pilots and the controllers were overall positive.

Work is continuing to refine and improve the concept. There is current work at looking to combine continuous descent approaches (CDA) with spacing to maintain capacity while greatly increasing fuel efficiency and noise reduction. Additional work will look at including limited maneuvering such as tromboning and fanning to allow the aircraft to overcome significant spacing deviation. Such situations might arise from the unexpected appearance of an arrival, a runway reassignment or reincorporating a missed approach. The flight crew could modify the "standard" profile to account for a gross spacing error and then use that as the basis for continual fine tuning using speed as in the current operation.

\section{References}

\footnotetext{
${ }^{1}$ Credeur, L. Basic Analysis of Terminal Operation Benefits Resulting from Reduced Vortex Separation Minima. NASA/TM-78624, 1977.

${ }^{2}$ Abbott, T. S. and G. C. Moen, Effect of Display Size on Utilization of Traffic Situation Display for Self-Spacing Task. NASA/TP-1885, 1981.

${ }^{3}$ Abbott, T. S. Simulation of a Cockpit Display for Increased Airport Capacity. Presented at the 27th Annual Meeting of the Human Factors Society. In Proceedings, Volume II, p.777-781, 1983.

${ }^{4}$ Williams, D. H. Time-Based Self-Spacing Techniques Using Cockpit Display of Traffic Information During Approach to Landing in a Terminal Area Vectoring Environment., NASA/TM-84601, 1983.

${ }^{5}$ Kelly, J. R. and T. S. Abbott, In-Trail Spacing Dynamics of Multiple CDTI-Equipped Aircraft Queues. NASA/TM85699, 1984 .

${ }^{6}$ Williams, D. H. and D. C. Wells Jet Transport Flight Operations Using Cockpit Display of Traffic Information During Instrument Meteorological Conditions, Simulation Evaluation., NASA/TP-2567, 1986.

${ }^{7}$ Credeur, L., J. A. Houck, W. R. Capron, and G. W. Lohr Delivery Performance of Conventional Aircraft by Terminal Area, Time-Based Air Traffic Control, A Real-time Simulation Evaluation. NASA/TP-2978, 1990.

${ }^{8}$ Abbott, T. S. A Compensatory Algorithm for the Slow-Down Effect on Constant-Time-Separation Approaches. NASA/TM-4285, September 1991.

${ }^{9}$ Sorensen, J. A., et al Traffic Alert and Collision Avoidance System (TCAS) - Cockpit Display of Traffic Information (CDTI) Investigation. DOT/FAA/RD-91/8, April 1991.

${ }^{10}$ Wang, G. and J. Hammer, Analysis of an Approach Spacing Application. 4th USA/Europe ATM2001 R\&D Seminar, Sante Fe, NM, USA, Dec. 3 - 7, 2001.

${ }^{11}$ Oseguera-Lohr, R. M., G. W.Lohr, T. S. Abbott, and T. M. Eischeid, Evaluation Of Operational Procedures For Using A Time-Based Airborne Interarrival Spacing Tool AIAA-2002-5824.
} 
${ }^{12}$ Oseguera-Lohr, R. M. and E. Nadler. Effects of an Approach Spacing Flight Deck Tool on Pilot Eye Scan. NASA/TM2004-212987.

${ }^{13}$ Lohr, G. W., R. M. Oseguera-Lohr, T. S. Abbott, and W. R. Capron, Flight Evaluation of a Time-Based Airborne Interarrival Spacing Tool. 5th EUROCONTROL / FAA ATM R\&D Seminar, Budapest, Hungary, June 23-27, 2003.

${ }^{14}$ Grimaud,I., E. Hoffman, L. Rognin, K. Zeghal, Spacing Instructions In Approach: Benefits And Limits From An Air Traffic Controller Perspective, AIAA 2004-5105, Reston, VA.

${ }^{15}$ Hoffman, E., D. Ivanescu, C. Shaw, K. Zeghal, Analysis of Constant Time Delay Airborne spacing Between Aircraft of Mixed Types in Varying Wind Conditions, Paper 77, Proceedings of the $5^{\text {th }}$ USA/Europe ATM Seminar at Budapest, Hungary, http://atm2003.eurocontrol.fr

${ }^{16}$ Abbott, T. S. Speed Control Law for Precision Terminal Area In-Trail Self Spacing, NASA TM 2002-211742, July 2002.

${ }^{17}$ Peters, M. E., M. G. Ballin and J. S. Sakosky. A Multi-Operator Simulation for Investigation of Distributed Air Traffic Management Concepts. AIAA Guidance, Navigation, and Control Conference \& Exhibit, Monterey, California, AIAA 2002-4596, August 5-8, 2002.

${ }^{18}$ Palmer, M. and M. Ballin, A High-Performance Simulated On-Board Avionics Architecture To Support Traffic Operations Research, AIAA Modeling and Simulation Technologies Conference, Austin, TX, AIAA 2003-5452, August 2003.

${ }^{19}$ Barmore, B.E., T.S. Abbott, K. Krishnamurthy, Airborne-Managed Spacing in Multiple Arrival Streams, Proceedings of the 24th Congress of the International Council of Aeronautical Sciences, 2004, Stockholm, Sweden, ICAS Secretariat

${ }^{20}$ Krishnamurthy, K., B. Barmore, F. Bussink, Airborne Precision Spacing In Merging Terminal Arrival Routes: A Fast-Time Simulation Study, Paper 76, Proceedings of the $6^{\text {th }}$ USA/Europe ATM Seminar at Baltimore, USA, http://atm2005.eurocontrol.fr 